\title{
Repeated columns and an old chestnut
}

\author{
R.P. Anstee* \\ Mathematics Department \\ The University of British Columbia \\ Vancouver, B.C. Canada V6T 1Z2 \\ anstee@math.ubc.ca
}

\author{
Linyuan $\mathrm{Lu}^{\dagger}$ \\ Mathematics Department \\ The University of South Carolina \\ Columbia, SC, USA \\ lu@math.sc.edu
}

Submitted: May 3, 2013; Accepted: Oct 7, 2013; Published: Oct 14, 2013

Mathematics Subject Classifications: 05D05

\begin{abstract}
Let $t \geqslant 1$ be a given integer. Let $\mathcal{F}$ be a family of subsets of $[m]=\{1,2, \ldots, m\}$. Assume that for every pair of disjoint sets $S, T \subset[m]$ with $|S|=|T|=k$, there do not exist $2 t$ sets in $\mathcal{F}$ where $t$ subsets of $\mathcal{F}$ contain $S$ and are disjoint from $T$ and $t$ subsets of $\mathcal{F}$ contain $T$ and are disjoint from $S$. We show that $|\mathcal{F}|$ is $O\left(m^{k}\right)$.

Our main new ingredient is allowing, during the inductive proof, multisets of subsets of $[m]$ where the multiplicity of a given set is bounded by $t-1$. We use a strong stability result of Anstee and Keevash. This is further evidence for a conjecture of Anstee and Sali. These problems can be stated in the language of matrices. Let $t \cdot M$ denote $t$ copies of the matrix $M$ concatenated together. We have established the conjecture for those configurations $t \cdot F$ for any $k \times 2$ $(0,1)$-matrix $F$.
\end{abstract}

Keywords: extremal set theory, extremal hypergraphs, (0,1)-matrices, multiset, forbidden configurations, trace, subhypergraph.

\section{Introduction}

We will be considering a problem in extremal hypergraphs that can be phrased as how many edges a hypergraph on $m$ vertices can have when there is a forbidden subhypergraph. There are a variety of ways to define this problem (we could, but do not, restrict to (simple) $k$-uniform hypergraphs). We can encode a hypergraph on $m$ vertices as an $m$-rowed $(0,1)$-matrix where the $i$ th column is the incidence vector of the $i$ th hyperedge.

${ }^{*}$ Research supported in part by NSERC, work done while visiting the second author at USC.

† This author was supported in part by NSF grant DMS 1000475 and DMS 1300547. 
A hypergraph is simple if there are no repeated edges. We define a matrix to be simple if it is a $(0,1)$-matrix with no repeated columns. We will use the language of matrices in this paper.

Let $M$ be an $m$-rowed (0,1)-matrix. Some notation about repeated columns is needed. For an $m \times 1(0,1)$-column $\alpha$, we define $\mu(\alpha, M)$ as the multiplicity of column $\alpha$ in a matrix $M$. We consider matrices of bounded column multiplicity. We define a matrix $A$ to be $t$-simple if it is a (0,1)-matrix and every column $\alpha$ of $A$ has $\mu(\alpha, A) \leqslant t$. Simple matrices are 1-simple. For a given matrix $M$, let $\operatorname{supp}(M)$ denote the maximal simple $m$-rowed submatrix of $M$, so that if $\mu(\alpha, M) \geqslant 1$ then $\mu(\alpha, \operatorname{supp}(M))=1$. The matrices below are a 3 -simple matrix $M$ and its $\operatorname{support} \operatorname{supp}(M)$.

$$
M=\left[\begin{array}{llllll}
0 & 1 & 0 & 1 & 1 & 0 \\
1 & 0 & 1 & 1 & 0 & 1
\end{array}\right], \quad \operatorname{supp}(M)=\left[\begin{array}{lll}
0 & 1 & 1 \\
1 & 0 & 1
\end{array}\right]
$$

For two $(0,1)$-matrices $F$ and $A$, we say that $F$ is a configuration in $A$, and write $F \prec A$ if there is a row and column permutation of $F$ which is a submatrix of $A$. Let $\mathcal{F}$ denote a finite set of $(0,1)$-matrices. Let $\operatorname{Avoid}(m, \mathcal{F}, t)$ denote all $m$-rowed $t$-simple matrices $A$ for which $F \nprec A$ for all $F \in \mathcal{F}$. We are most interested in cases with $|\mathcal{F}|=1[5]$. We do not require any $F \in \mathcal{F}$ to be simple which is quite different from usual forbidden subhypergraph problems. Let $\|A\|$ denote the number of columns of $A$. Our extremal function of interest is

$$
\operatorname{forb}(m, \mathcal{F})=\max _{A}\{\|A\|: A \in \operatorname{Avoid}(m, \mathcal{F}, 1)\}
$$

We find it helpful to also define

$$
\operatorname{forb}(m, \mathcal{F}, t)=\max _{A}\{\|A\|: A \in \operatorname{Avoid}(m, \mathcal{F}, t)\}
$$

If $A \in \operatorname{Avoid}(m, \mathcal{F}, t)$ then $\operatorname{supp}(A) \in \operatorname{Avoid}(m, \mathcal{F}, 1)$ and $\|A\| \leqslant t \cdot\|\operatorname{supp}(A)\|$. We obtain

$$
\operatorname{forb}(m, \mathcal{F}) \leqslant \operatorname{forb}(m, \mathcal{F}, t) \leqslant t \cdot \text { forb }(m, \mathcal{F}),
$$

so that the asymptotic growth of $\operatorname{forb}(m, \mathcal{F})$ is the same as that of $\operatorname{forb}(m, \mathcal{F}, t)$ for fixed $t$.

We have an important conjecture about forb $(m, F)$. We use the notation $[M \mid N]$ to denote the matrix obtained from concatenating the two matrices $M$ and $N$. We use the notation $k \cdot M$ to denote the matrix $[M|M| \cdots \mid M]$ consisting of $k$ copies of $M$ concatenated together. Let $I_{k}$ denote the $k \times k$ identity matrix and let $I_{k}^{c}$ denote the $(0,1)$-complement of $I_{k}$. Let $T_{k}$ denote the $k \times k$ triangular $(0,1)$-matrix with the $(i, j)$ entry being 1 if and only if $i \leqslant j$. For an $m_{1} \times n_{1}$ matrix $X$ and an $m_{2} \times n_{2}$ matrix $Y$, we define the 2-fold product $X \times Y$ as the $\left(m_{1}+m_{2}\right) \times n_{1} n_{2}$ matrix where each column consisting of a column of $X$ placed on a column of $Y$ and this is done in all possible ways. This extends to $p$-fold products. 
Definition 1. Let $X(F)$ be the smallest $p$ so that $F \prec A_{1} \times A_{2} \times \cdots \times A_{p}$ for every choice of $A_{i}$ as either $I_{m / p}, I_{m / p}^{c}$ or $T_{m / p}$ for sufficiently large $m$.

Alternatively, assuming $F \nprec I$ or $F \nprec I^{c}$ or $F \nprec T$, then $X(F)-1$ is the largest choice of $p$ so that $F \nprec A_{1} \times A_{2} \times \cdots \times A_{p}$ for some choices of $A_{i}$ as either $I_{m / p}, I_{m / p}^{c}$ or $T_{m / p}$. We note that if $A_{1} \times A_{2} \times \cdots \times A_{p} \in \operatorname{Avoid}(m, F)$, then forb $(m, F)$ is $\Omega\left(m^{p}\right)$.

Details are in [5]. We are assuming $m$ is large and divisible by $p$, in particular that $m \geqslant(k+1)(k \ell+1)$ so that $m / p \geqslant k \ell+1$. Divisibility by $p$ does not affect the asymptotic growth, thus forb $(m, F)$ is $\Omega\left(m^{X(F)-1}\right)$ using an appropriate $(X(F)-1)$-fold product.

Conjecture 2. [4] Let $F$ be given. Then forb $(m, F)=\Theta\left(m^{X(F)-1}\right)$.

The conjecture was known to be true for all 3-rowed $F$ [4] and all $k \times 2 F$ [3]. Section 3 shows how Theorem 3 establishes the conjecture for matrices $t \cdot F$ when $F$ is a $k \times 2$ matrix. It is of interest to generalize Conjecture 2 to $\operatorname{forb}(m, \mathcal{F})$ where $|\mathcal{F}|>1$ but we know example of $\mathcal{F}$ where the modified form of the conjecture fails (see [5]).

We define $F_{e, f, g, h}$ as the $(e+f+g+h) \times 2$ matrix consisting of $e$ rows [1 1], $f$ rows [1 0], $g$ rows [0 1] and $h$ rows [0 0]. Let $\mathbf{1}_{e} \mathbf{0}_{f}$ denote the $(e+f) \times 1$ vector of $e$ 1's on top of $f$ 0's so that $F_{e, f, g, h}=\left[\mathbf{1}_{e+f} \mathbf{0}_{g+h} \mid \mathbf{1}_{e} \mathbf{0}_{f} \mathbf{1}_{g} \mathbf{0}_{h}\right]$. We let $\mathbf{1}_{e}$ denote the $e \times 1$ vector of $e$ 1 's and $\mathbf{0}_{f}$ denote the $f \times 1$ vector of $f 0$ 's. Our main result is the following which had foiled many previous attempts.

Theorem 3. Let $t \geqslant 2$ be given. Then forb $\left(m, t \cdot F_{0, k, k, 0}\right)$ is $\Theta\left(m^{k}\right)$.

The forbidden configuration $t \cdot F_{0, k, k, 0}$ in the language of sets, consists of two disjoint $k$-sets $S, T$, and a family of $t$ sets containing $S$ but disjoint from $T$, and the other family of another $t$ sets containing $T$ but disjoint from $S$. This theorem echoes our statement in the abstract.

The result for $t=2$ and $k=2$ was proven in [1] and many details worked out for $t=2$ and $k>2$ by the first author and Peter Keevash. The extension for $t>2, k=2$ had been open since then [5]. The proof for $t>2, k=2$ is in Section 2. The proof for $t>2, k>2$ is in Section 3. Matrices $F_{6}(t), F_{7}(t)$ were given in [5] as 4-rowed forbidden configurations (with some columns of multiplicity $t$ ) for which Conjecture 2 predicts forb $\left(m, F_{6}(t)\right)$ and forb $\left(m, F_{7}(t)\right)$ are $O\left(m^{2}\right)$. Note that $t \cdot F_{0,2,2,0} \prec F_{6}(t)$ and $t \cdot F_{0,2,2,0} \prec F_{7}(t)$ and so Theorem 3 is a step towards these bounds which would establish Conjecture 2 for all 4-rowed $F$. Our proof use a new induction given in Section 2 that considers $t$-simple matrices as well as a strong stability result Lemma 10. We offer some additional applications in Section 4.

\section{New Induction}

We consider a new form of the standard induction for forbidden configurations [5]. Let $F$ be a matrix with maximum column multiplicity $t$. Thus $F \prec t \cdot \operatorname{supp}(F)$. Let 
$A \in \operatorname{Avoid}(m, F, t-1)$. Assume $\|A\|=\operatorname{forb}(m, \mathcal{F}, t-1)$. Given a row $r$ we permute rows and columns of $A$ to obtain

$$
A=\operatorname{row} r \rightarrow\left[\begin{array}{ccc}
0 & 0 \cdots & 11 \cdots \\
G & H
\end{array}\right]
$$

Now $\mu(\alpha, G) \leqslant t-1$ and $\mu(\alpha, H) \leqslant t-1$. For those $\alpha$ for which $\mu(\alpha,[G H]) \geqslant t$, let $C$ be formed with $\mu(\alpha, C)=\min \{\mu(\alpha, G), \mu(\alpha, H)\}$. We rewrite our decomposition of $A$ as follows:

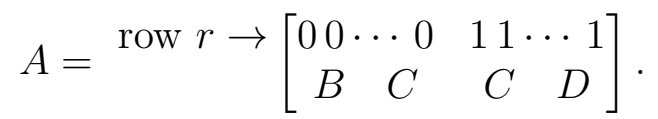

Then we deduce that $[B C D]$ and $C$ are both $(t-1)$-simple. The former follows from $\mu(\alpha,[B C D])=\mu(\alpha, G)+\mu(\alpha, H)-\min \{\mu(\alpha, G), \mu(\alpha, H)\} \leqslant t-1$. We have that $F \nprec[B C D]$ for $F \in \mathcal{F}$. Also for any $F^{\prime} \prec C$ then $[01] \times F^{\prime} \prec A$ so we define

$$
\mathcal{G}=\left\{F^{\prime}: \text { for } F \in \mathcal{F}, F \prec[01] \times F^{\prime} \text { and } F \nprec[01] \times F^{\prime \prime} \text { for all } F^{\prime \prime} \prec F^{\prime}, F^{\prime \prime} \neq F^{\prime}\right\} \text {. }
$$

Basically, $\mathcal{G}$ is the family after removing redundancy from all configurations $F^{\prime}$ that are obtained by removing one row from any $F$ in $\mathcal{F}$.

Also since each column $\alpha$ of $C$ has $\mu(\alpha,[G H]) \geqslant t$, we deduce that $\operatorname{supp}(F) \nprec C$ for each $F \in \mathcal{F}$. Our induction on $m$ becomes:

$$
\begin{aligned}
& \operatorname{forb}(m, \mathcal{F}, t-1)=\|A\|=\|[B C D]\|+\|C\| \\
& \quad \leqslant \operatorname{forb}(m-1, \mathcal{F}, t-1)+(t-1) \cdot \operatorname{forb}(m-1, \mathcal{G} \cup\{\operatorname{supp}(F): F \in \mathcal{F}\}) .
\end{aligned}
$$

Lemma 4. Let $H$ be a given simple matrix satisfying forb $(m, H)$ is $O\left(m^{\ell}\right)$. Then forb $(m, t \cdot H)$ is $O\left(m^{\ell+1}\right)$.

Proof. We use the induction (5) where $F=t \cdot H$ and $H=\operatorname{supp}(F)$. Induction on $m$ yields the desired bound.

Proof of Theorem 3 for $k=2$ : We will use induction on $m$ to show forb $(m, t$. $\left.F_{0,2,2,0}, t\right)$ is $O\left(m^{2}\right)$. The maximum multiplicity of a column in $t \cdot F_{0,2,2,0}$ is $t$ and $F_{0,2,2,0}=$ $\operatorname{supp}\left(t \cdot F_{0,2,2,0}\right)$. Also $t \cdot F_{0,2,2,0} \prec[01] \times\left(t \cdot F_{0,2,1,0}\right)$. Let $A \in \operatorname{Avoid}\left(m, t \cdot F_{0,2,2,0}, t-1\right)$ with $\|A\|=\operatorname{forb}\left(m, t \cdot F_{0,2,2,0}, t-1\right)$. Apply (5). We have

$$
\begin{aligned}
& \operatorname{forb}\left(m, t \cdot F_{0,2,2,0}, t-1\right)=\|A\|=\|[B C D]\|+\|C\| \\
& \quad \leqslant \operatorname{forb}\left(m-1, t \cdot F_{0,2,2,0}, t-1\right)+(t-1) \cdot \operatorname{forb}\left(m-1,\left\{F_{0,2,2,0}, t \cdot F_{0,2,1,0}\right\}\right) .
\end{aligned}
$$

We apply Lemma 5 with induction on $m$ to deduce that forb $\left(m, t \cdot F_{0,2,2,0}, t-1\right)$ is $O\left(m^{2}\right)$. Then by (1), forb $\left(m, t \cdot F_{0,2,2,0}\right)$ is also $O\left(m^{2}\right)$.

Theorem 3 was proven for $t=k=2$ in [1] using induction in the spirit of (5) $((t-1)$-simple matrices are simple) and Lemma 5 for $t=2$. 
Lemma 5. We have that forb $\left(m,\left\{F_{0,2,2,0}, t \cdot F_{0,2,1,0}\right\}\right)$ is $O(m)$.

Proof. Let $A \in \operatorname{Avoid}\left(m,\left\{F_{0,2,2,0}, t \cdot F_{0,2,1,0}\right\}\right)$. Avoiding $F_{0,2,2,0}$ creates structure: Let $X_{i}$ denote the columns of $A$ of column sum $i$. Let $J_{a \times b}$ denote the $a \times b$ matrix of 1 's and let $0_{a \times b}$ denote the $a \times b$ matrix of 0 's. Now $F_{0,2,2,0} \nprec X_{i}$ and so for $\left\|X_{i}\right\| \geqslant 3$, we may deduce that there is a partition of the rows $[m]$ into $A_{i} \cup B_{i} \cup C_{i}$. Let $x_{i}=\left|X_{i}\right|$. After suitable row and column permutations, we have $X_{i}$ as follows:

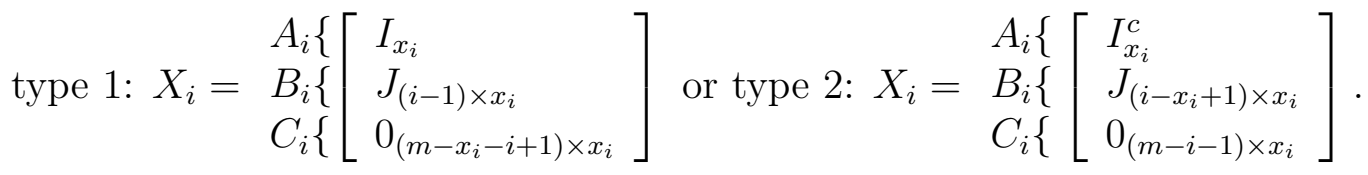

We will say $i$ is of type $j(j=1$ or $j=2)$ if the columns of sum $i$ are of type $j$. These are the sunflowers (for type 1) and inverse sunflowers (type 2) of [7] where for type 1 the petals are $A_{i}$ with center $B_{i}$.

Let $T(1)=\left\{i: i\right.$ is of type 1 and $\left.\left\|X_{i}\right\| \geqslant t+2\right\}$. We wish to show for that $B_{i} \subset B_{j}$ for $i, j \in T(1)$ and $i<j$. Assume $p \in B_{i} \backslash B_{j}$. Given that $\left|B_{i}\right|<\left|B_{j}\right|$, there are two rows $r, s \in B_{j} \backslash B_{i}$. Then we find a copy of $t \cdot F_{0,2,1,0}$ in rows $p, r, s$ of $\left[X_{i} X_{j}\right]$ (we would not choose the possible column of $X_{i}$ that has a 1 in row $r$ and the column of $X_{i}$ that has a 1 in row $s$ ), a contradiction showing no such $p$ exists and hence $B_{i} \subset B_{j}$.

We form a matrix $Y_{1}$ from those $X_{i}$ with $i \in T(1)$. We have $\left\|Y_{1}\right\|=\sum_{i \in T(1)}\left\|X_{i}\right\|=$ $\sum_{i \in T(1)}\left|A_{i}\right|$. Assume $\sum_{i \in T(1)}\left|A_{i}\right|>(t+1) m$. Then there is some row $p$ and $(t+2)$ set $\{s(1), s(2), \ldots, s(t+2)\}$ with $p \in A_{i}$ for all $i \in\{s(1), s(2), \ldots, s(t+2)\}$. Assume $s(1)<s(2)<\cdots<s(t+2)$. We have $B_{s(1)} \subset B_{s(2)} \subset \cdots \subset B_{s(t+2)}$. We may choose $r, s \in B_{s(t+2)} \backslash B_{s(t)}$ so that $r, s \in A_{s(i)} \cup C_{s(i)}$ for $i=1,2, \ldots, t$. We find a copy of $t \cdot F_{0,2,1,0}$ in rows $p, r, s$ as follows. We take one column from each $X_{s(j)}$ for $j=1,2, \ldots, t$ and $t$ columns from the $X_{s(t+2)}$. We conclude that $\left\|Y_{1}\right\| \leqslant(t+1) m$. Similarly the matrix $Y_{2}$ formed from those $X_{i}$ such that $i$ is of type 2 and $\left\|X_{i}\right\| \geqslant t+2$ has $\left\|Y_{2}\right\| \leqslant(t+1) m$. Now $Y_{1}$ and $Y_{2}$ represent all columns of $A$ with the exception of columns of sum $i$ with $\left\|X_{i}\right\| \leqslant t+1$ and so we conclude $\|A\| \leqslant\left\|Y_{1}\right\|+\left\|Y_{2}\right\|+(t+1)(m-1)+2$. Thus $\|A\|$ is $O(m)$.

\section{More evidence for the Conjecture}

This section first explores the Conjecture 2 for $t \cdot F$ when $F$ is $k \times 2$. The section concludes with the proof of Theorem 3 for $k>2$. The following verifies Conjecture 2 for all $k \times 2 F$. Note that any $k \times 2$ matrix $F$ can be written as $F_{a, b, c, d}(b \geqslant c)$ under proper row and column permutations. Since forb $(m, F)$ is invariant under taking $(0,1)$ complement, we can further assume $a \geqslant d$. The case of $t=1$ was solved in [3] by the following theorem.

Theorem 6. [3] Suppose $a \geqslant d$ and $b \geqslant c$. Then forb $\left(m, F_{a, b, c, d}\right)$ is $\Theta\left(m^{a+b-1}\right)$ if either $b>c$ or $a, b \geqslant 1$. Also forb $\left(m, F_{a, 0,0, d}\right)$ is $\Theta\left(m^{a}\right)$ and forb $\left(m, F_{0, b, b, 0}\right)$ is $\Theta\left(m^{b}\right)$. 
Note that Conjecture 2 is verified if there is a product construction avoiding $F$ yielding the same asymptotic growth as an upper bound on forb $(m, F)$. The $k$-fold product $I_{m / k} \times I_{m / k} \times \cdots \times I_{m / k} \in \operatorname{Avoid}\left(m, t \cdot F_{0, k, k, 0}\right)$ has $\Theta\left(m^{k}\right)$ columns. Thus Theorem 3 verifies the conjecture for $t \cdot F_{0, k, k, 0}$. The following results verify the conjecture for $t \cdot F$ for the remaining $k \times 2 F$.

Theorem 7. For $b>c$ or $a, b \geqslant 1$ then forb $\left(m, t \cdot F_{a, b, c, d}\right)$ is $\Theta\left(m^{a+b}\right)$.

Proof. The upper bound follows from forb $\left(m, F_{a, b, c, d}\right)$ being $\Theta\left(m^{a+b-1}\right)$ and then applying Lemma 4 . The lower bound follows from $2 \cdot \mathbf{1}_{a+b} \prec t \cdot F_{a, b, c, d}$ so that the $(a+b)$-fold product $I_{m /(a+b)} \times I_{m /(a+b)} \times \cdots \times I_{m /(a+b)} \in \operatorname{Avoid}\left(m, F_{a, b, c, d}\right)$ and hence forb $\left(m, t \cdot F_{a, b, c, d}\right)$ is $\Omega\left(m^{a+b}\right)$.

Theorem 8. Let $a \geqslant d$ be given. Then forb $\left(m, t \cdot F_{a, 0,0, d}\right)$ is $\Theta\left(m^{a}\right)$.

Proof. This follows using Lemma 9 repeatedly and also forb $\left(m, t \cdot F_{a, 0,0,0}\right)$ is $O\left(m^{a}\right)$ using Theorem 14. The $a$-fold product $I_{m / a} \times I_{m / a} \times \cdots \times I_{m / a} \in \operatorname{Avoid}\left(m, t \cdot F_{a, 0,0, d}\right)$.

The following result can be found in the survey on forbidden configurations [5]

Lemma 9. Assume forb $(m, F)$ is $O\left(m^{\ell}\right)$. Then forb $\left(m,\left[\begin{array}{l}1 \\ 0\end{array}\right] \times F\right)$ is $O\left(m^{\ell+1}\right)$.

Here is the summary of results on forb $\left(m, t \cdot F_{a, b, c, d}\right)(a \geqslant d$ and $b \geqslant c)$, which verify Conjecture 2 for all $k \times 2 F$.

\begin{tabular}{|c|c|c|c|c|}
\hline$t$ & Configuration & result & reference & Lower bound construction \\
\hline \multirow{3}{*}{$t=1$} & $\begin{array}{l}F_{a, b, c, d}(b>c \\
\text { or } a, b \geqslant 1)\end{array}$ & $\Theta\left(m^{a+b-1}\right)$ & {$[3]$} & $\overbrace{I \times I \times \cdots I \times I}^{a+b-1}$ \\
\hline & $F_{a, 0,0, d}$ & $\Theta\left(m^{a}\right)$ & {$[3]$} & $\overbrace{I \times I \times \cdots I \times I}$ \\
\hline & $F_{0, b, b, 0}$ & $\Theta\left(m^{b}\right)$ & {$[3]$} & $\overbrace{I \times I \times \cdots I \times I}^{b-1} \times T$ \\
\hline \multirow{3}{*}{$t \geqslant 2$} & $\begin{array}{c}t \cdot F_{a, b, c, d}(b>c \\
\text { or } a, b \geqslant 1)\end{array}$ & $\Theta\left(m^{a+b}\right)$ & Lemma 4 & $\overbrace{I \times I \times \cdots I \times I}^{a+b}$ \\
\hline & $t \cdot F_{a, 0,0, d}$ & $\Theta\left(m^{a}\right)$ & Lemma 4 & $\overbrace{I \times I \times \cdots I \times I}$ \\
\hline & $t \cdot F_{0, b, b, 0}$ & $\Theta\left(m^{b}\right)$ & Theorem 3 & $\overbrace{I \times I \times \cdots I \times I}^{b-1} \times T$ \\
\hline
\end{tabular}

Table 1: All cases of forb $\left(m, t \cdot F_{a, b, c, d}\right)$ with $a \geqslant d$ and $b \geqslant c$.

We note that the bound for $\operatorname{forb}\left(m, t \cdot F_{a, 0,0, d}\right)$ can be readily established by a pigeonhole argument. We return to Theorem 3 and first obtain some useful lemmas. Let 
$X_{i} \in \operatorname{Avoid}\left(m, F_{0, k, k, 0}\right)$ with all column sums $i$. We define $X_{i}$ to be of type $(a, b)$ if $a, b \geqslant 0$ are integers with $a+b=k-1$ and there is a partition $C_{i} \cup D_{i}=[m]$ with $\left|D_{i}\right|+a-b=i$ such that any column $\alpha$ of $X_{i}$ has exactly $a$ 1's in rows $C_{i}$ and exactly $b$ 0 's in rows $D_{i}$. We are able to use this structure in view of the following 'strong stability' result:

Lemma 10. [3] Let $Y_{i} \in \operatorname{Avoid}\left(m, F_{0, k, k, 0}\right)$ with all column sums $i$. Assume $\left\|Y_{i}\right\| \geqslant$ $(6(k-1))^{5 k+2} m^{k-2}$. Then there is an $m$-rowed submatrix $X_{i}$ of $Y_{i}$ and a pair of integers $a, b \geqslant 0$ with $a+b=k-1$ such that $X_{i}$ is of type $(a, b)$ and where $\left\|Y_{i}\right\|-\left\|X_{i}\right\| \leqslant m^{k-3}$.

Lemma 11. Let $X_{i} \in \operatorname{Avoid}\left(m, F_{0, k, k, 0}\right)$ have all columns of sum $i$ and assume $X_{i}$ is of type $(a, b)$ with $a, b \geqslant 1$ with $a+b=k-1$. Let $C_{i} \cup D_{i}=[m]$ be the associated partition of the rows. We form a bipartite graph $G_{i}=\left(V_{i}, E_{i}\right)$ with $V_{i}=\left(\begin{array}{c}C_{i} \\ a\end{array}\right) \cup\left(\begin{array}{c}D_{i} \\ b\end{array}\right)$ where we have $(C, D) \in E_{i}$ if there is a column of $X_{i}$ with a 1's in rows $C$ and $D_{i} \backslash D$ and $b$ O's in rows $D$ and $C_{i} \backslash C$. Assume $\left|E_{i}\right| \geqslant 2 \mathrm{~km}^{k-2}$. Then there is subgraph $G_{i}^{\prime}=\left(V_{i}^{\prime}, E_{i}^{\prime}\right)$ of $G_{i}$ with $\left|E^{\prime}\right| \geqslant \frac{1}{2}\left|E_{i}\right|$ such that for every pair $C \in\left(\begin{array}{c}C_{i} \\ a\end{array}\right)$ and $D \in\left(\begin{array}{c}D_{i} \\ b\end{array}\right)$ with $(C, D) \in E^{\prime}$ we have

$$
d_{G_{i}^{\prime}}(C) \geqslant(b+1 / 2) m^{b-1}, \quad d_{G_{i}^{\prime}}(D) \geqslant(a+1 / 2) m^{a-1} .
$$

Proof. Simply delete vertices $C \in\left(\begin{array}{c}C_{i} \\ a\end{array}\right)$ with $d_{G}(C)<(b+1 / 2) m^{b-1}$ and vertices $D \in$ $\left(\begin{array}{c}D_{i} \\ b\end{array}\right)$ with $d_{G}(D)<(a+1 / 2) m^{a-1}$ and continue deleting vertices until conditions $(6)$ are satisfied for any remaining vertices of $G^{\prime}$. This will delete a maximum of $(b+$ $1 / 2) m^{b-1}\left(\begin{array}{c}\left|C_{i}\right| \\ a\end{array}\right)+(a+1 / 2) m^{a-1}\left(\begin{array}{c}\left|D_{i}\right| \\ b\end{array}\right)<k m^{k-2}$ edges which deletes less than half the edges of $G$.

Lemma 12. Let $k$ be given. Then forb $\left(m,\left\{F_{0, k, k, 0}, t \cdot F_{0, k, k-1,0}\right\}\right)$ is $O\left(m^{k-1}\right)$.

Proof. Let $A \in \operatorname{Avoid}\left(m,\left\{F_{0, k, k, 0}, t \cdot F_{0, k, k-1,0}\right\}\right)$. Let $Y_{i}$ denote the columns of $A$ of column sum $i$. For all $i$ for which $\left|Y_{i}\right|<(6(k-1))^{5 k+2} m^{k-2}$, delete the columns of $Y_{i}$ from $A$. This may delete $(6(k-1))^{5 k+2} m^{k-1}$ columns. For $i$ with $\left|Y_{i}\right| \geqslant(6(k-1))^{5 k+2} m^{k-2}$, apply Lemma 10 and obtain $X_{i}$ with $\left|X_{i}\right| \geqslant(6(k-1))^{5 k+2} m^{k-2}-m^{k-3}$.

We consider a choice $a, b$ with $a+b=k-1$. Let $T(a, b)=\left\{i: X_{i}\right.$ is of type $\left.(a, b)\right\}$. We will show that $\sum_{i \in T(a, b)}\left|X_{i}\right| \leqslant(t k) m^{k-1}$.

Case 1. $a, b \geqslant 1$.

Create $G_{i}$ as described in Lemma 11 to obtain $G_{i}^{\prime}$ for each $i \in T(a, b)$. Now if $\sum_{i \in T(a, b)}\left|E_{i}^{\prime}\right|>(t+1) m^{a+b}$, then there will be some edge $(C, D) \in E_{i}^{\prime}$ for at least $t+2$ choices $i \in T(a, b)$. Let those choices be $s(1), s(2), \ldots, s(t+2)$ where $s(1)<s(2)<$ $\cdots<s(t+2)$. We wish to show that $X_{s(i)}$ has $t \cdot F_{0, k-1,0,0}$ on rows $C \cup D$.

$$
\begin{aligned}
& \operatorname{rows} C\left\{\begin{array}{lll}
1 & \overbrace{11 \cdots 1}^{t} & \overbrace{00 \cdots 0}^{t} \\
1 & 11 \cdots 1 & 00 \cdots 0 \\
1 & 11 \cdots 1 & 00 \cdots 0
\end{array}\right. \\
& \operatorname{rows} D\left\{\begin{array}{lll}
0 & 11 \cdots 1 & 00 \cdots 0 \\
0 & 11 \cdots 1 & 00 \cdots 0
\end{array}\right.
\end{aligned}
$$


For a given set $D \in\left(\begin{array}{c}D_{s(i)} \\ b\end{array}\right)$, we compute $\left|\left\{H \in\left(\begin{array}{c}D_{s(i)} \\ b\end{array}\right): H \cap D \neq \emptyset\right\}\right| \leqslant \sum_{j=1}^{b}\left(\begin{array}{c}b \\ j\end{array}\right)\left(\begin{array}{c}D_{s(i)} \backslash D \\ b-j\end{array}\right)<$ $b m^{b-1}$.

Now if $d_{G^{\prime}}(C) \geqslant(b+1 / 2) m^{b-1}$ and $(C, D) \in E_{s(i)}^{\prime}$ then there are at least $t$ edges $(C, H) \in E_{s(i)}^{\prime}$ with $H \cap D=\emptyset$. We are using $(b+1 / 2) m^{b-1}>b m^{b-1}+t+2$ which is true for $m$ large enough and so asymptotics are unaffected. Thus we have $t$ columns of $X_{s(1)}$ with $\mathbf{1}_{k-1}$ on rows $C \cup D$ and, because these columns have a 1's on rows $C \subseteq C_{s(1)}$, these columns are 0's on the remaining rows of $C_{s(1)} \backslash C$.

Similarly, because $d_{G_{i}^{\prime}}(D) \geqslant(a+1 / 2) m^{a-1}$ there will be $t+2$ edges $(K, D) \in E_{s(i)}$ with $K \cap C=\emptyset$ and so there are $t$ columns of $X_{s(t+2)}$ with $\mathbf{0}_{k-1}$ on rows $C \cup D$ and, because these columns have 0's on rows $D$, these columns are 1's on rows of $D_{s(t+2)} \backslash D$.

We choose $k$ rows in $Z=D_{s(t+2)} \backslash D_{s(1)}$ so that $Z \subseteq C_{s(1)}$. We deduce that in the chosen $t$ columns of $X_{s(1)}$ we have $\mathbf{0}_{k}$ in rows $Z$ since $Z \subseteq C_{s(1)} \backslash C$ and the columns have $\mathbf{1}_{k-1}$ in rows $C \cup D$. In the chosen $t$ columns of $X_{s(t+2)}$ we have $\mathbf{1}_{k}$ in rows $Z$ since $Z \subset D_{s(t+2)} \backslash D$ and the columns have $\mathbf{0}_{k-1}$ in rows $C \cup D$. This yields $t \cdot F_{0, k, k-1,0}$, a contradiction. Thus $\sum_{i \in T y p e(a, b)}\left|E_{i}^{\prime}\right| \leqslant(t+1) m^{k-1}$. This concludes Case 1 .

Case 2. $a=k-1, b=0$ or $a=0, b=k-1$.

We proceed similarly. We need only consider $a=k-1, b=0$ since the case $a=0, b=$ $k-1$ is just the $(0,1)$-complement. For $i \in T(k-1,0), X_{i}$ has partition $C_{i} \cup D_{i}=[m]$ and columns of $X_{i}$ have 1's on exactly $k-1$ rows of $C_{i}$ and all 1's on rows $D_{i}$. Assume $\sum_{i \in T(k-1,0)}\left|X_{i}\right| \geqslant(t k) m^{k-1}$. Then there are $t k$ choices $s(1), s(2), \ldots, s(t k) \in T(k-1,0)$ where $s(1)<s(2)<\cdots<s(t k)$ such that, for some $C \in\left(\begin{array}{c}C_{s}(i) \\ k-1\end{array}\right)$, each $X_{s(i)}$ has a column with 1's in rows $C \cup D_{s(i)}$ and 0's in rows $C_{s(i)} \backslash C$. We wish to find $t \cdot F_{0, k-1,0,0}$ in $A$ in rows $C$ as follows using one column from each of $X_{s(i)}$ for $i=1,2, \ldots, t$ and $t$ columns from $X_{s(t k)}$.

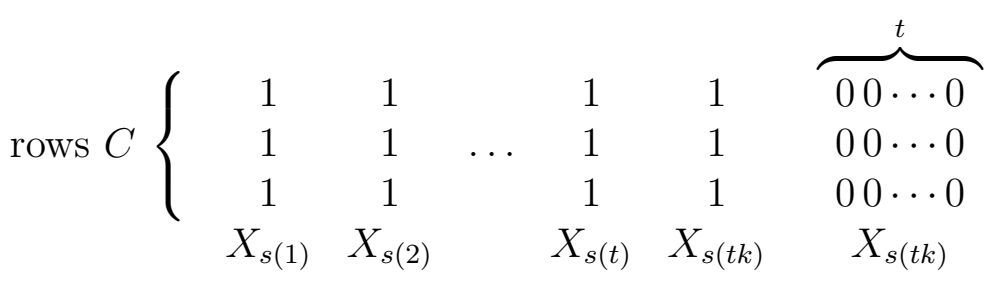

Given our choice $C \in\left(\begin{array}{c}C_{s(t k)} \\ k-1\end{array}\right)$, we compute that $\left|\left\{K \in\left(\begin{array}{c}C_{s(k t)} \\ k-1\end{array}\right): K \cap C \neq \emptyset\right\}\right|<k m^{k-2}$. Thus with $\left|X_{s(k t)}\right| \geqslant k m^{k-2}$, there will be $t$ choices $K_{1}, K_{2}, \ldots, K_{t}$ disjoint from $C$ and hence one column of $X_{s(k t)}$ for each $i=1,2, \ldots, t$ with $\mathbf{1}_{k-1}$ on rows of $K_{i} \subseteq C_{s(k t)} \backslash C$ and 0 's on $C_{s(k t)} \backslash K_{i}$ and hence $\mathbf{0}_{k-1}$ on rows $C$.

We will show below that we can choose $D \subset D_{s(k t)} \backslash \cup_{i=1}^{t} D_{s(i)}$ with $|D|=k$. Then we can find $t \cdot F_{0, k, k-1,0}$ as follows. We have one column in $X_{s(i)}$ for each $i=1,2, \ldots, t$ which is $\mathbf{1}_{k-1}$ on rows $C$ and $\mathbf{0}_{k}$ on rows $D$ (since $D \subset C_{s(i)} \backslash C$ for each $i=1,2, \ldots, t$ ). The $t$ columns of $X_{s(t k)}$ we have selected have $\mathbf{0}_{k-1}$ on rows $C$ and 1 's on $D_{s(k t)}$ where $D \subseteq D_{s(k t)}$ and hence $\mathbf{1}_{k}$ on rows $D$. These $2 t$ columns yield $t \cdot F_{0, k, k-1,0}$ in $\left[X_{s(1)}\left|X_{s(2)} \cdots\right| X_{s(t)} \mid X_{s(k t)}\right]$. 
To show that $D$ can be chosen we first show that $D_{s(i)} \backslash D_{s(j)} \leqslant k-2$ for $s(i)<s(j)$. Assume the contrary, $D_{s(i)} \backslash D_{s(j)} \geqslant k-1$ for $s(i)<s(j)$. We choose $C^{\prime} \subseteq D_{s(i)} \backslash D_{s(j)}$ with $\left|C^{\prime}\right|=k-1$. Given $s(j)>s(i)$, then $D_{s(j)} \backslash D_{s(i)} \geqslant k$ and so we may choose $D^{\prime} \subseteq D_{s(j)} \backslash D_{s(i)}$ with $\left|D^{\prime}\right|=k$. Now $C^{\prime} \subset C_{s(j)}$ and $D^{\prime} \subset C_{s(i)}$. The number of possible columns of $X_{s(j)}$ with at least one 1 on the rows $C^{\prime}$ is at most $m^{k-2}$ and with $\left|X_{s(j)}\right| \geqslant m^{k-1}+t$, we find $t$ columns of $X_{s(j)}$ with 0's on rows $C^{\prime}$ and necessarily with 1's on rows $D^{\prime}$. The number of possible columns of $X_{s(i)}$ with at least one 1 on the rows of $D^{\prime}$ is $\left|D^{\prime}\right| m^{k-2}<m^{k-1}$. Given $\left|X_{s(i)}\right| \geqslant m^{k-1}+t$, we find $t$ columns of $X_{s(i)}$ with 0 's on rows $D^{\prime}$ and necessarily with 1 's on rows $C^{\prime}$. This yields $t \cdot F_{0, k, k-1,0}$ in $\left[X_{s(i)} \mid X_{s(j)}\right]$, a contradiction. Thus $D_{s(i)} \backslash D_{s(j)} \leqslant k-2$ for $s(i)<s(j)$. We may now conclude that $\left|D_{s(k t} \backslash \cup_{i=1}^{t} D_{s(i)}\right| \geqslant k$ and so a choice for $D$ exists. We conclude $\sum_{i \in T(k-1,0)}\left|X_{i}\right| \leqslant(t k) m^{k-1}$. This concludes Case 2 .

There are $k+1$ choices for type $(a, b)$ and so

$$
\sum_{i=0}^{m}\left|X_{i}\right| \leqslant \sum_{j=0}^{k}\left(\sum_{i \in T(j, k-1-j)}\left|X_{i}\right|\right) \leqslant(k+1)(2 t k) m^{k-1}
$$

and so $\|A\| \leqslant(2 t k(k+1)) m^{k-1}+(6(k-1))^{5 k+2} m^{k-2}$ which is $O\left(m^{k-1}\right)$.

Proof of Theorem 3 for $k \geqslant 3$ : We use (5) so that forb $\left(m, t \cdot F_{0, k, k, 0}, t-1\right) \leqslant \operatorname{forb}\left(m-1, t \cdot F_{0, k, k, 0}, t-1\right)+(t-1)$ forb $\left(m,\left\{F_{0, k, k, 0}, t \cdot F_{0, k, k-1,0}\right\}\right)$.

Induction on $m$ and Lemma 12 yields the bound.

\section{Some applications of the Induction}

Let $K_{k}$ denote the $k \times 2^{k}$ of all possible $(0,1)$-columns on $k$ rows. The following is the fundamental result about forbidden configurations.

Theorem 13. [Sauer [10], Perles and Shelah [11], Vapnik and Chervonenkis [12]] We have that

$$
f o r b\left(m, K_{k}\right)=\left(\begin{array}{c}
m \\
k-1
\end{array}\right)+\left(\begin{array}{c}
m \\
k-2
\end{array}\right)+\cdots+\left(\begin{array}{c}
m \\
0
\end{array}\right) .
$$

Thus forb $\left(m, K_{k}\right)$ is $\Theta\left(m^{k-1}\right)$.

We can apply this result as follows.

Theorem 14. [8] Let $F$ be a given $k \times \ell(0,1)$-matrix. Then forb $(m, F)$ is $O\left(m^{k}\right)$.

Proof. Let $t$ be the maximum multiplicity of a column in $F$ (of course $t \leqslant \ell$ ). Then $F \prec t \cdot K_{k}$ and $\operatorname{so} \operatorname{supp}(F) \prec K_{k}$. Now Lemma 4 combined with Theorem 13 yields the result. 
Interestingly this yields the exact result for forb $\left(m, 2 \cdot K_{k}\right)[9]$. A more precise result of Anstee and Füredi [2] for forb $\left(m, t \cdot K_{k}\right)$ has the leading term being bounded by $\frac{t+k-1}{k+1}\left(\begin{array}{c}m \\ k\end{array}\right)$ for $t \geqslant 2$. The following surprising result was obtained by Balogh and Bollobás.

Theorem 15. [6] Let $k$ be given. There is a constant $c_{k}$ with forb $\left(m,\left\{I_{k}, I_{k}^{c}, T_{k}\right\}\right)=c_{k}$.

This yields the following.

Theorem 16. Let $t, k \geqslant 2$ be given. Then forb $\left(m,\left\{t \cdot I_{k}, t \cdot I_{k}^{c}, t \cdot T_{k}\right\}\right)$ is $\Theta(m)$.

Proof. Apply Lemma 4. The matrix $I_{m} \in \operatorname{Avoid}\left(m,\left\{t \cdot I_{k}, t \cdot I_{k}^{c}, t \cdot T_{k}\right\}\right)$ shows that forb $\left(m,\left\{t \cdot I_{k}, t \cdot I_{k}^{c}, t \cdot T_{k}\right\}\right)$ is $\Theta(m)$.

Lemma 4 is interesting for those $H$ for which forb $(m, H)$ is $O\left(m^{\ell}\right)$ and the number of rows in $H$ is bigger than $\ell$ (see [5] for examples). It is not expected that this will resolve any boundary cases, namely those $F$ for which forb $(m,[F \mid \alpha])$ is bigger than forb $(m, F)$ by a linear factor (or more) for all choices $\alpha$ which are either not present in $F$ or occur at most once in $F$. The previously mentioned $F_{6}(t)$ and $F_{7}(t)$ have quite complicated structure and the induction (5) does not appear to work directly.

\section{References}

[1] R.P. Anstee, Some Problems Concerning Forbidden Configurations, preprint from 1990.

[2] R.P. Anstee and Z. Füredi, Forbidden submatrices, Discrete Math. 62 (1986), 225243.

[3] R.P. Anstee and P. Keevash, Pairwise intersections and forbidden configurations, European J. of Combin. 27 (2006), 1235-1248.

[4] R.P. Anstee, A. Sali, Small Forbidden Configurations IV, Combinatorica 25(2005), 503-518.

[5] R.P. Anstee, A Survey of forbidden configurations results, Elec. J. Combinatorics 20 (2013), DS20, 56pp.

[6] J. Balogh, B. Bollobás, Unavoidable Traces of Set Systems, Combinatorica, 25 (2005), 633-643.

[7] P. Frankl, Z. Füredi and J. Pach, Bounding one-way differences, Graphs and Combinatorics, 3 (1987), 341-347.

[8] Z. Füredi, personal communication, 1983.

[9] H.O.F. Gronau, An extremal set problem, Studia Sci.Math. Hungar. 15(1980), 29-30.

[10] N. Sauer, On the density of families of sets, J. Combinatorial Theory Ser. A 13 (1972), 145-147. 
[11] S. Shelah, A combinatorial problem: Stability and order for models and theories in infinitary languages, Pacific J. Mathematics 4 (1972), 247-261.

[12] V.N. Vapnik and A.Ya. Chervonenkis, On the uniform convergence of relative frequencies of events to their probabilities, Theory of Probability and Applications 25 (1971), 264-280. 